\title{
Molecular Interaction Process
}

National Cancer Institute

\section{Source}

National Cancer Institute. Molecular Interaction Process. NCI Thesaurus. Code C18219.

Involves temporary, non-covalent binding of two or more molecules as a result of intermolecular physical forces and often involves spatial complementarity between the interacting objects. 